\title{
Video Endoscopic Inguinal Lymphadenectomy in Post Saphenous Vein Graft Harvest (Coronary Artery Bypass) Status
}

\author{
(D) Anandan Murugesan, (D) Ramesh Chinnasamy \\ Kovai Medical Center and Hospital, Department of Urology, Tamil Nadu, India
}

\begin{abstract}
Video endoscopic inguinal lymphadenectomy (VEIL), either by the classical or robot-assisted approach, is performed to manage inguinal lymph nodes in penile carcinoma. Coronary artery bypass graft (CABG) is common in the age group affected by penile carcinoma. Saphenous vein graft harvesting (SVGH) is done for CABG and may be associated with scarred subcutaneous planes hindering VEIL. A 65-year-old male was diagnosed with T2 high-grade penile carcinoma. He had undergone CABG eight years ago. He underwent robot-assisted VEIL. The initial subcutaneous space beneath Camper's fascia was created by finger dissection and further developed by balloon inflation. A small space was made with difficulty to insert the ports. Monopolar shears were used to make a sharp dissection to develop the subcutaneous plane, and scarring was present close to Camper's fascia. Multiple small venules were visible and managed with bipolar diathermy. The rest of the procedure was performed as usual. The console time and estimated blood loss were higher in the SVGH limb. The drain output was higher on the SVGH side. Both sides had six negative lymph nodes. Minimal skin duskiness was noted on the ipsilateral side, which healed without any sequelae. This is the first documented report of robot-assisted VEIL, post saphenous vein harvest for CABG. It is associated with a slightly longer procedure time and more blood loss, but a satisfactory oncological outcome. This report highlights the feasibility and safety of robot-assisted VEIL in post-CABG saphenous vein harvest status.
\end{abstract}

Keywords: Penile carcinoma, video endoscopic inguinal lymphadenectomy, coronary artery bypass graft

\section{Introduction}

The prognosis of penile carcinoma is dependent to a fair extent on lymph node management. Open approach by either classical Dressler's or modified Catalona's inguinal lymphadenectomy is the standard treatment. However, inguinal lymphadenectomy is associated with considerable morbidity, especially skin loss and lymphedema (1). Video endoscopic inguinal lymphadenectomy (VEIL) helps to mitigate some complications (2). Saphenous vein graft harvest (SVGH) is usually done for coronary artery bypass graft (CABG) (3). This involves dissection in the subcutaneous space where we dissect for VEIL. With CABG being increasingly performed, a patient who had CABG presenting for VEIL is not uncommon. The potential problems caused by the previous SVGH in performing VEIL are unknown.

\section{Case Report}

A 65-year-old male was diagnosed with T2 high-grade penile carcinoma and underwent a partial penectomy.
Magnetic resonance imaging did not reveal any significant lymphadenopathy. He had undergone CABG eight years ago with SVGH in his right lower limb (Figure 1). He was scheduled for an inguinal lymphadenectomy one month after penectomy. He underwent robot-assisted VEIL.

Under general anesthesia, he was placed in the supine position, with his lower limb abducted and externally rotated (frogleg position). The landmarks were marked initially, and a 1.5 $\mathrm{cm}$ incision was made $3 \mathrm{~cm}$ distal to the apex of the femoral triangle. The initial subcutaneous space beneath Camper's fascia was created by finger dissection and further developed by balloon inflation (Figure 2a). A small space was made with difficulty, especially medially, to insert the four ports. The robot was docked from the left shoulder aspect.

Monopolar shears were used to make a sharp dissection to develop the plane below Camper's fascia. Multiple small venules were visible and managed with bipolar diathermy (Figure 2b). The dissection was extended until the predetermined lateral and medial borders of dissection as per Dressler's quadrilateral were

Correspondence: Anandan Murugesan MS, MCh, Kovai Medical Center and Hospital, Department of Urology, Tamil Nadu, India Phone: +04224324642 E-mail: anandan79@gmail.com ORCID-ID: orcid.org/0000-0003-2691-2115

Received: 23.03.2021 Accepted: 25.04.2021

Cite this article as: Murugesan A, Chinnasamy R. Video Endoscopic Inguinal Lymphadenectomy Post Saphenous Vein Graft Harvest (Coronary Artery Bypass) Status. J Urol Surg 2021;8(4):294-296.

๑Copyright 2021 by the Association of Urological Surgery / Journal of Urological Surgery published by Galenos Publishing House. 


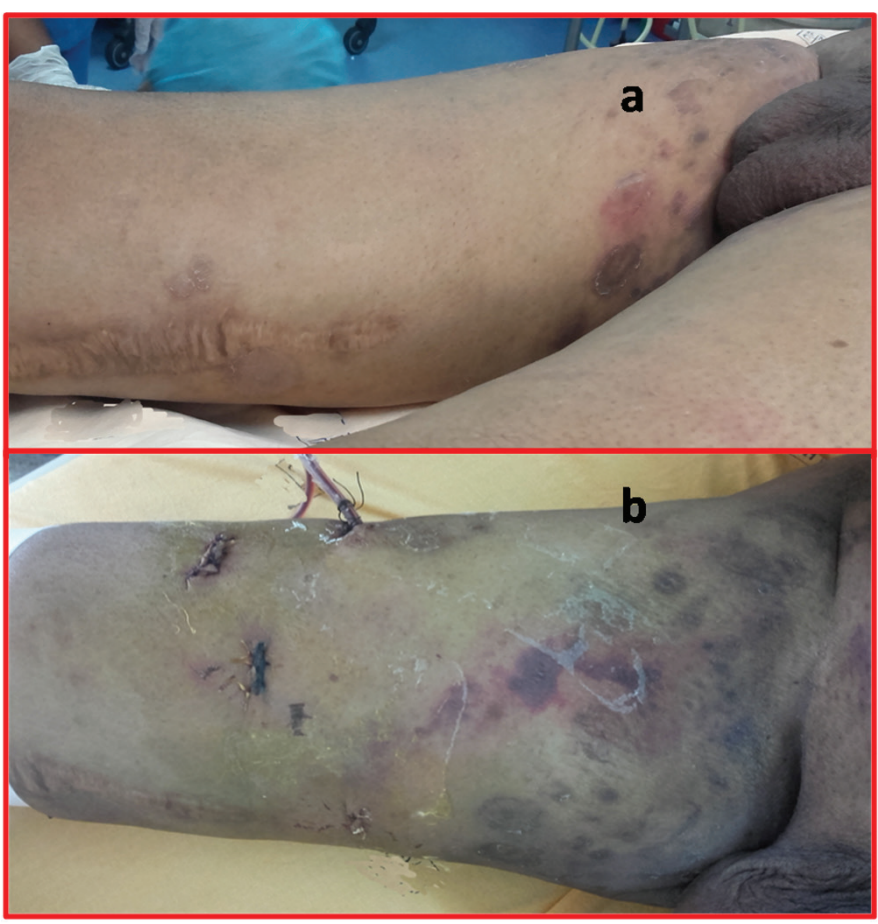

Figure 1. a) Preoperative image, b) Postoperative image

reached. Superiorly fascial dissection was done till the external oblique aponeurosis was visualized. The fibrofatty tissue was then dissected off the muscle fascia in a distal to proximal manner. This plane was relatively well preserved, as expected (Figure 2c). A small remnant of the saphenous vein of about 5 $\mathrm{cm}$ was identified and it was traced proximally until the femoral vein. Fibrofatty tissues around the femoral vessels were dissected out. The specimen was removed through the camera port after placing it in a retrieval bag. The procedure was repeated on the left side similarly, with the robot docked on the right side.

The console time and estimated blood loss were 140 minutes and $150 \mathrm{~mL}$ on the right (SVGH) side and 110 minutes and $50 \mathrm{~mL}$ on the left side. He was mobilized during the day with elastic compression stockings. His drain output was more on the SVH side and for a longer duration. His drain was removed on postoperative day (POD) 12 on the SVGH side compared with POD5 on the other side. Six lymph nodes were retrieved on both sides, and all were negative for the tumor. Minimal skin duskiness was noted on the SVGH side, which healed without any sequelae. Bilateral minimal lymphedema was noted at oneyear follow-up.

\section{Discussion}

VEIL, either classical or robot-assisted, is preferred over open if expertise is available. Wound-related complications are decreased using this approach (2). An essential step in VEIL is the development of tissue planes. Similar to retroperitoneal
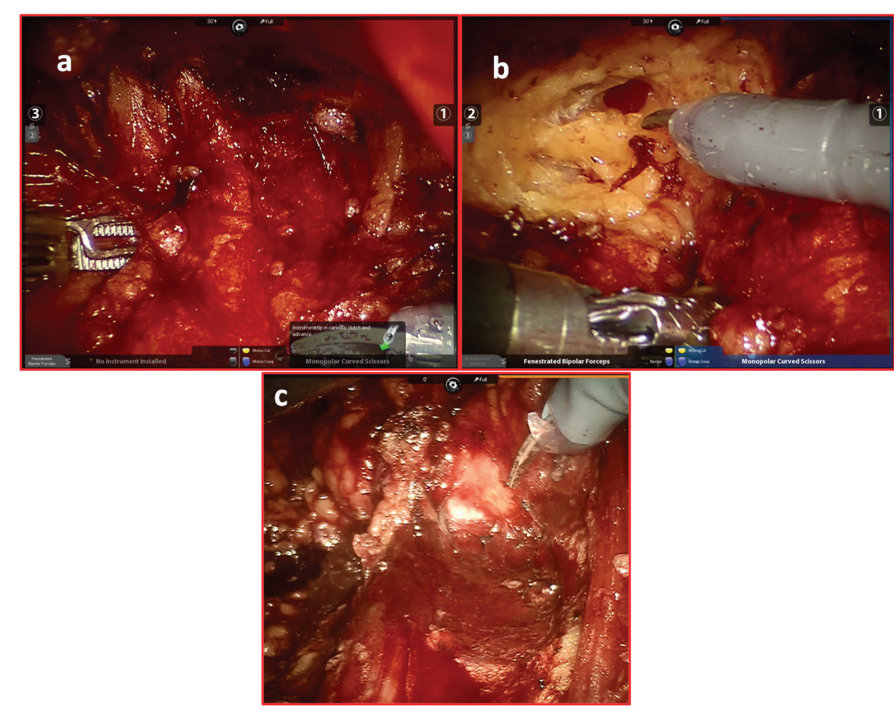

Figure 2. a) Initial view after balloon dilatation, b) Plane developed by sharp dissection with endoshears with coagulation of bleeders, c) Well preserved deep planes

planes during retroperitoneoscopy, previous surgeries in those areas and resultant scars might hinder tissue space development $(4,5)$. SVGH is performed during CABG (3). Now, it is common to have patients who necessitate inguinal lymphadenectomy postCABG. SVGH is done with either a single contiguous incision or multiple small incisions. There have been no previous reports on VEIL among those with previous SVH.

We experienced some difficulty in developing the tissue planes, especially on the medial aspect. After placing the initial ports and minimal dissection, additional tissue planes needed sharp dissections with monopolar shears. Usually, simple balloon dilatation will be sufficient to create tissue spaces. However, due to the fibrous scarring in these tissue spaces, sharp dissection was needed. Moreover, bleeding was comparatively more do the dilated small venules. Fibrotic tissue planes may cause flap thinning, resulting in later necrosis. The fascial planes near the muscles were relatively well preserved. The console time was more on the SVGH side due to more fibrous tissues and more bleeding encountered from small venules.

Saphenous vein preservation is one of the components of Catalona's modification to prevent lymphedema (1). However, we did not find increased lymphedema on the side of SVGH. Skin necrosis was also not seen.

Open inguinal lumphadenectomy is associated with high morbidity and complication rates of up to $50 \%$. VEIL has a better complication profile, especially regarding skin flap necrosis. The operative time, lymph node yield, and blood loss were comparable to the open lymphadenectomy in a large series (6).

This is the first report of robot-assisted VEIL in a patient who has undergone previous SVGH. It emphasizes that it is oncologically 
safe and technically feasible, though the procedure incurs more blood loss and increased console time.

Robot-assisted VEIL is a feasible and oncologically safe approach even in patients who have undergone SVGH for CABG.

\section{Ethics}

Informed Consent: This case report is presented after obtaining informed consent from the patient.

Peer-review: Externally peer-reviewed.

\section{Authorship Contributions}

Surgical and Medical Practices: A.M., R.C., Concept: A.M., Design: A.M., Data Collection or Processing: A.M., R.C., Analysis or Interpretation: A.M., Literature Search: A.M., R.C., Writing: A.M.

Conflict of Interest: No conflict of interest was declared by the authors.

Financial Disclosure: The authors declared that this study received no financial support.

\section{References}

1. Protzel C, Alcaraz A, Horenblas S, Pizzocaro G, Zlotta A, Hakenberg OW. Lymphadenectomy in the surgical management of penile cancer. Eur Urol 2009;55:1075-1088.

2. Pahwa HS, Misra S, Kumar A, Kumar V, Agarwal A, Srivastava R. Video Endoscopic Inguinal Lymphadenectomy (VEIL)--a prospective critical perioperative assessment of feasibility and morbidity with points of technique in penile carcinoma. World J Surg Oncol 2013;11:42.

3. Caliskan E, de Souza DR, Böning A, Liakopoulos OJ, Choi YH, Pepper J, Gibson CM, Perrault LP, Wolf RK, Kim KB, Emmert MY. Saphenous vein grafts in contemporary coronary artery bypass graft surgery. Nat Rev Cardiol 2020;17:155-169.

4. Quintela RS, Cotta LR, Neves MF, Abelha DLJr, Tavora JE. Retroperitoneoscopic nephrectomy in benign pathology. Int Braz J Urol 2006;32:521-528.

5. Liapis D, de la Taille A, Ploussard G, Robert G, Bastien L, Hoznek A, Vordos D, Abbou C, Salomon L. Analysis of complications from 600 retroperitoneoscopic procedures of the upper urinary tract during the last 10 years. World J Urol 2008;26:523-530.

6. Kumar V, Sethia KK. Prospective study comparing video-endoscopic radical inguinal lymph node dissection (VEILND) with open radical ILND (OILND) for penile cancer over an 8-year period. BJU Int 2017;119:530-534. 